\title{
GAMMA-AMINOBUTYRIC ACID (GABA) ENCEPHALITIS: CASE REPORT
}

Mohd Farid bin Md Yusof ${ }^{1}$, Shalisah binti Sharip ${ }^{1}$, Suriati binti Mohamed Saini ${ }^{1}$, Fairuz Nazri binti Abdul Rahman ${ }^{1}$, Hamid bin Abdul Rahman ${ }^{1}$ and Raihanah binti Abdul Khalid ${ }^{2}$

${ }^{1}$ Psychiatry Department, Hospital Canselor Tuanku Muhriz, Universiti Kebangsaan Malaysia, Jalan Yaacob Latif, Bandar Tun Razak, Cheras, Kuala Lumpur, Malaysia, ${ }^{2}$ Assunta Hospital, Jalan Templer, Petaling Jaya, Selangor, Malaysia

Presenter: Mohd Farid bin Md Yusof, blackjeack27@yahoo.com

A Severe encephalitis with an uncontrolled seizure may produce impairment in a variety of cognitive functions. The appropriate treatment for cognitive impairment postencephalitisis are currently unknown. We are reporting a case of depression and cognitive impairment post- encephalitis with an uncontrolled seizure. Our case presented with global cognitive impairment and severe depression. The patient showed improvement following pharmacological treatments coupled with cognitive stimulation, and family education. The combination of multimodal treatments are needed following encephalitis. Intensive cognitive training may serve as a potential harbor for treatment of cognitive impairment post-encephalitis. 